\title{
T- Cell Lymphoblastic Lymphoma/Leukemia: A Case Report
}

\author{
Cristabel Robles Hidalgo*, Evette Yassa and Jean Pratt Daniel \\ Department of Internal Medicine Lincoln Medical Center, USA \\ *Corresponding author: Cristabel Robles Hidalgo, Department of Internal Medicine Lincoln Medical Center, USA
}

\begin{tabular}{|c|c|}
\hline ARTICLE INFO & ABSTRACT \\
\hline Received: 幽 August 27, 2019 & Citation: Cristabel Robles H, Evette Y, Jean Pratt D. Cristabel Robles Hidalgo, Department \\
\hline Published: September 03, 2019 & $\begin{array}{l}\text { of Internal Medicine Lincoln Medical Center, USA. Biomed J Sci \& Tech Res 21(1)-2019. } \\
\text { BJSTR. MS.ID.003546. }\end{array}$ \\
\hline
\end{tabular}

\section{Introduction}

T-lymphoblastic leukemia/lymphoma (T-LBL) is rare, aggressive neoplasm of lymphoblasts committed to T-cell lineage involving bone marrow (BM), blood or presenting as a tissue-based mass involving the thymus, lymph nodes or extra nodal sites. Typically, T-LBL presents as an Advanced widely disseminated disease. Lymphoma is heralded by lymphadenopathy; we are presenting a case of a patient who presented with significant bilateral inguinal lymphadenopathy.

\section{Case Report}

39-year-old Nigerian male with no past medical history was referred by infectious disease department for Eosinophilic dyscrasia. Patient had presented to the ED with complaint of 1 month of intermittent fever, night sweats, chills, fatigue, loss of appetite with unintentional weight loss, weakness, malaise, and itching. The rest of ROS was negative, his most recent travel was 3 years ago, and he denied any sick contacts. He had lived in the Bronx for the last 3 years, and lives in Staten Island prior to that. He denied any hiking or camping recently. He initially visited the ED on August $29^{\text {th }}, 2018$ with a temperature of $102.7^{\circ} \mathrm{F}$ and all other vital signs within normal limits. Laboratory investigation at that time showed microcytic anemia with eosinophils and high reticulocyte count, suggestive of blood-borne parasite leading to consideration of malaria vs viral syndrome. Peripheral smear at that time was inconclusive. Additional labs findings were notable for thrombocytopenia, elevated LDH, low iron, decreased iron saturation, and decreased TIBC. Chest X-Ray showed no abnormalities and HIV test was negative. Initial differentials included Babesiosis, Malaria, Anaplasmosis, Lyme disease, EBV, and CMV. Empiric treatment was begun for Babesiosis with Atorvaquone and Azithromycin. He was instructed to monitor fever pattern and return in one week

The patient returned to the ED September 10th, 2018 with the same symptoms and was admitted to medicine. Laboratory investigation at which time showed neutropenia, worsening microcytic anemia, and increasing eosinophilia (28.6\%) with LDH of 304. Hepatic functional panel was within normal limits. Physical exam was significant only for palpable spleen and extensive bilateral inguinal lymphadenopathy. Abdominal and pelvic CT with contrast showed extensive lymphadenopathy of the inguinal region, pelvis, and para-aortic region bilaterally as well as splenomegaly and numerous small sclerotic foci involving vertebral bodies of lower thoracic spine concerning for metastatic disease. Admitted to medicine with fever and significant eosinophilia, investigations continued for various infectious processes Figure 1. During his course in the hospital, he had episodes of fever to $102^{\circ} \mathrm{F}$ daily to twice daily, which were treated with Tylenol. Benadryl was given for pruritus. Repeated thick and thin peripheral smear did not show parasites. Findings were positive for EBV IgG, CMV IgG, RMSF IgG, B. Quintana IgM, and Parvo B19 IgG. He was negative for syphilis, chlamydia, and gonorrhea, strongyloides, B. Burgdorferi, B. Henselae, B. Quintana, Coxiella burnetii, leptospira, RMSF IgM, Typhus, Monospot, CMV IgM, HBV, HCV, Parvo B19 IgM, and HTLV-I/ II. 


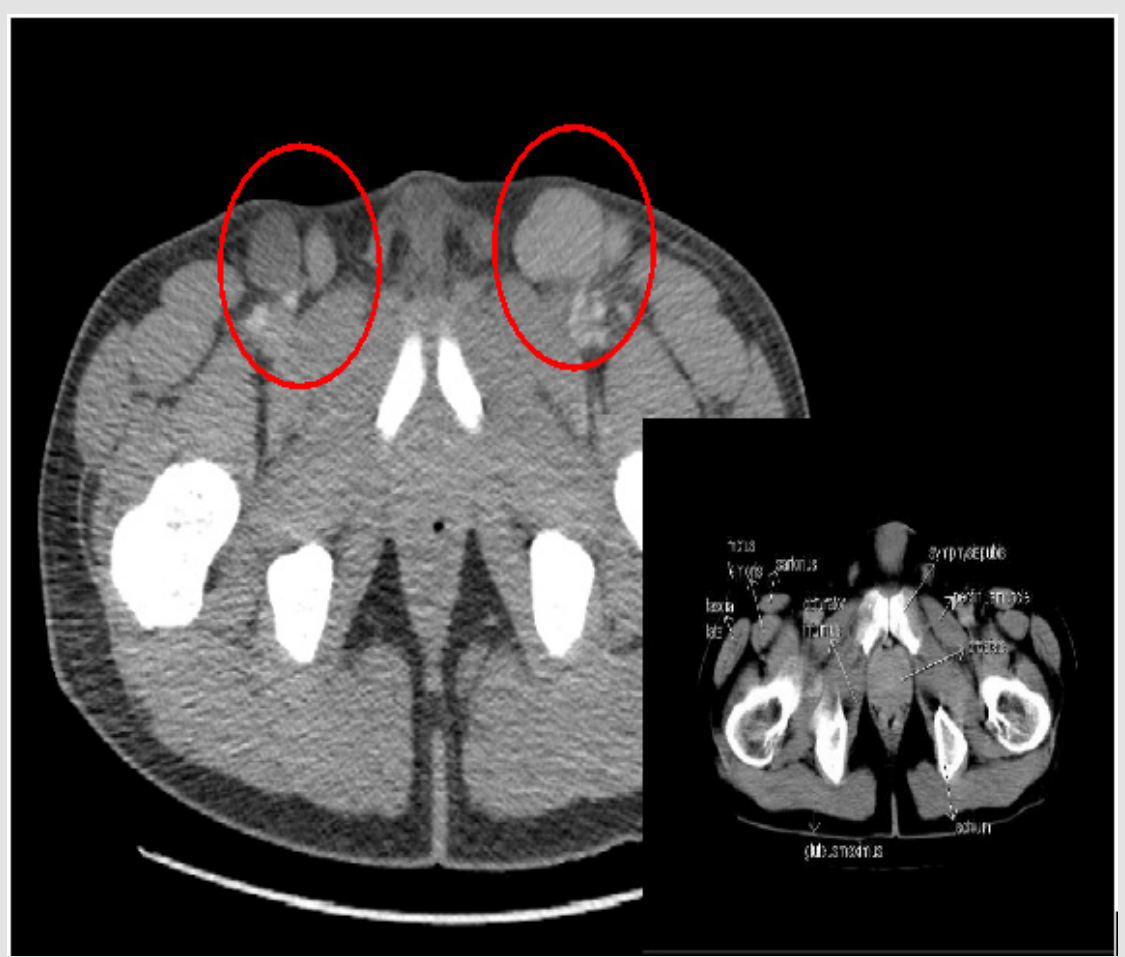

Figure 1.

The patient reported subjective improvement, and lymph node biopsy was scheduled for September $13^{\text {th }}, 2018$. Left inguinal lymph node biopsy results were positive for Ki-67 staining and showed expanded interfollicular area with many small lymphoid cells expressing CD2, CD3, CD5, CD7, CD4, CD8, and TdT+. Pathological impression was T-Lymphoblastic Lymphoma/Leukemia. Flow cytometry showed immature $\mathrm{T}$ cells also suggestive of $\mathrm{T}$ lymphoblastic lymphoma Figure 2. The patient was discharged with follow-up for bone marrow biopsy which was ultimately negative. He was referred to Montefiore Hospital for aggressive chemotherapy with Hyper fractionated CVAD (Cyclophosphamide, Vincristine, Adriamycin/Doxorubicin, and Dexamethasone). This treatment was discontinued after 2 cycles due to severe lower extremity neuropathy. He now follows with hematology department at Bellevue hospital for continue treatment with chemotherapy.

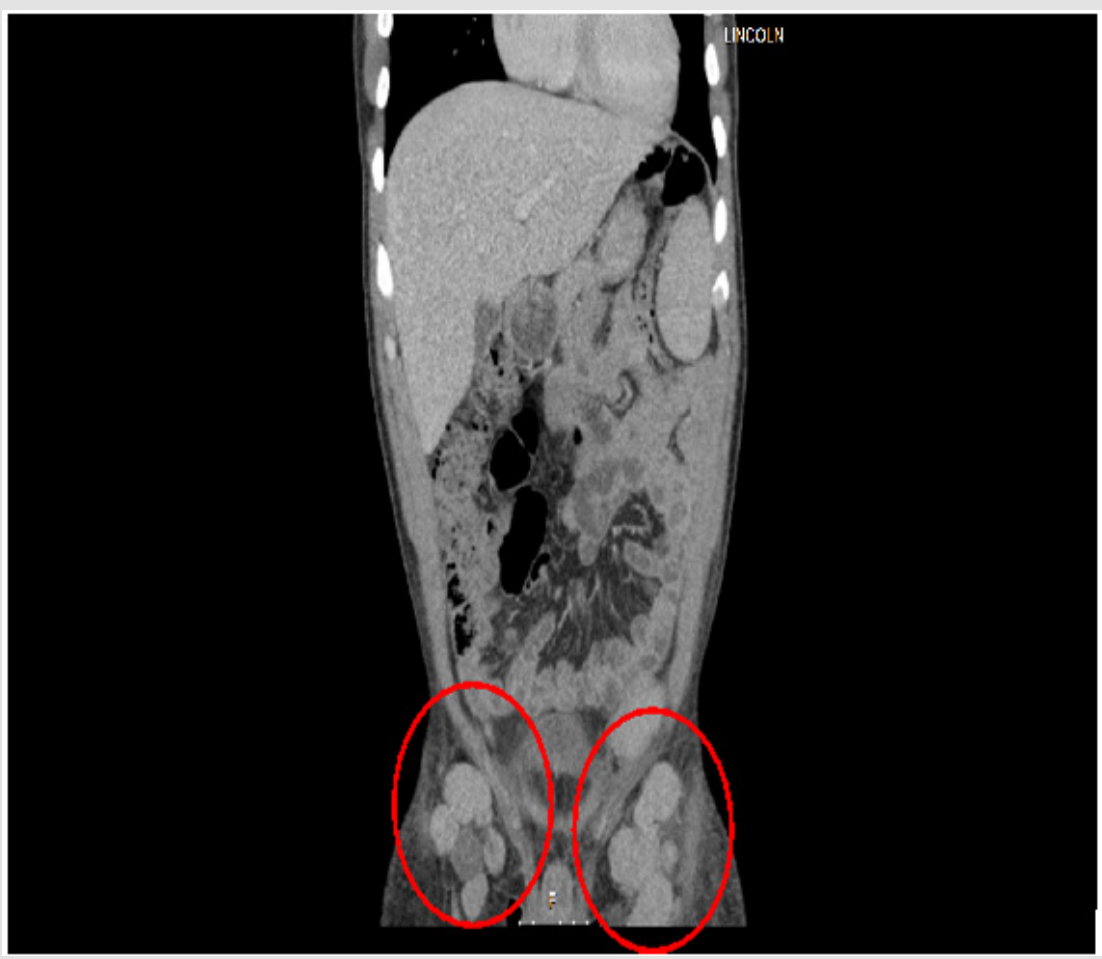

Figure 2. 


\section{Discussion}

Lymphoblastic lymphoma (LBL) is a rare disease, accounting for approximately $8 \%$ of all lymphoid malignancies. It occurs in males more often than females at a ratio of $1: 4$, and it is also most commonly diagnosed in children younger than 15 years old, with a rate of only 0.8 per 100,000 people aged 25 to 64 years old [1]. Studied risk factors for the development of the disease include exposure to ionizing radiation, infections, occupational exposures, and inherited susceptibility. The infection most strongly associated with acute T cell-LBL is Human T-cell Leukemia Virus-1 (HTLV-1), which is endemic to regions of Japan, the Caribbean, Africa, and South America, and which acts as a direct carcinogen. Additional infections of concern include HIV1 and Hepatitis $\mathrm{C}$ virus. With regards to occupational exposure, benzene and electromagnetic field exposure have shown a strong association with LBL development [1]. T-LBL is less common than T-cell Acute Lymphoblastic Leukemia (T-ALL) and is often defined by the confinement of the neoplasm within a mass with minimal blood or bone marrow involvement [2].

Diagnostic criteria include the presence of blast cells in peripheral blood smear, tissue biopsy, and/or bone marrow biopsy. In the peripheral smear the lymphoblasts are identified as small cells with scant cytoplasm and condensed nuclear chromatin, but may also be larger cells with ample cytoplasm, dispersed chromatin, and many nuclei. Tissue biopsy would show lymphoblasts in a diffuse, lymph node, or paracortical pattern. Though rare, it is also possible to see nodules that resemble follicular lymphoma with or without eosinophils infiltrating the lymph nodes. Histochemical analysis shows positive staining for TdT as a marker of lymphoid progenitor cell origin, with variable expression of CD1a, CD2, CD3, CD4, CD5, CD7, and CD8. While T-LBL and T-ALL are similar in many ways, T-LBL usually demonstrates a more mature immunophenotype [3].

Those with T-LBL are likely to present with cervical, supraclavicular, and axillary lymphadenopathy with or without mediastinal mass [4]. It is a clinically aggressive disease with patients usually presenting at stage IV with B symptoms such as fever, night sweats, and weight loss, and frequently with elevated LDH. At initial presentation bone marrow is usually normal but that does not preclude infiltration in a later stage Figure 3. The German Multicenter Trials for Adult Acute Lymphoblastic Leukemia (GMALL) showed the only significant prognostic factor for survival in T-LBL was elevated LDH, which was present in our patient. Multiple mutations have been identified to play a role in the development of T-LBL, including activating mutations in NOTCH1 (which encodes for transmembrane protein) or inactivating mutations of FBXW7 which negatively regulates NOTCH. Additionally, dysregulated expression of HOX11, TLX1, TAL1, LYL1, LM01, and LMO2 were associated with mutation arrest at known stages of normal thymic development [2]. The GMALL also showed that Notch/FBXW7 mutation (without RAS/PTEN mutation/ deletion) could be considered a favorable prognostic factor.

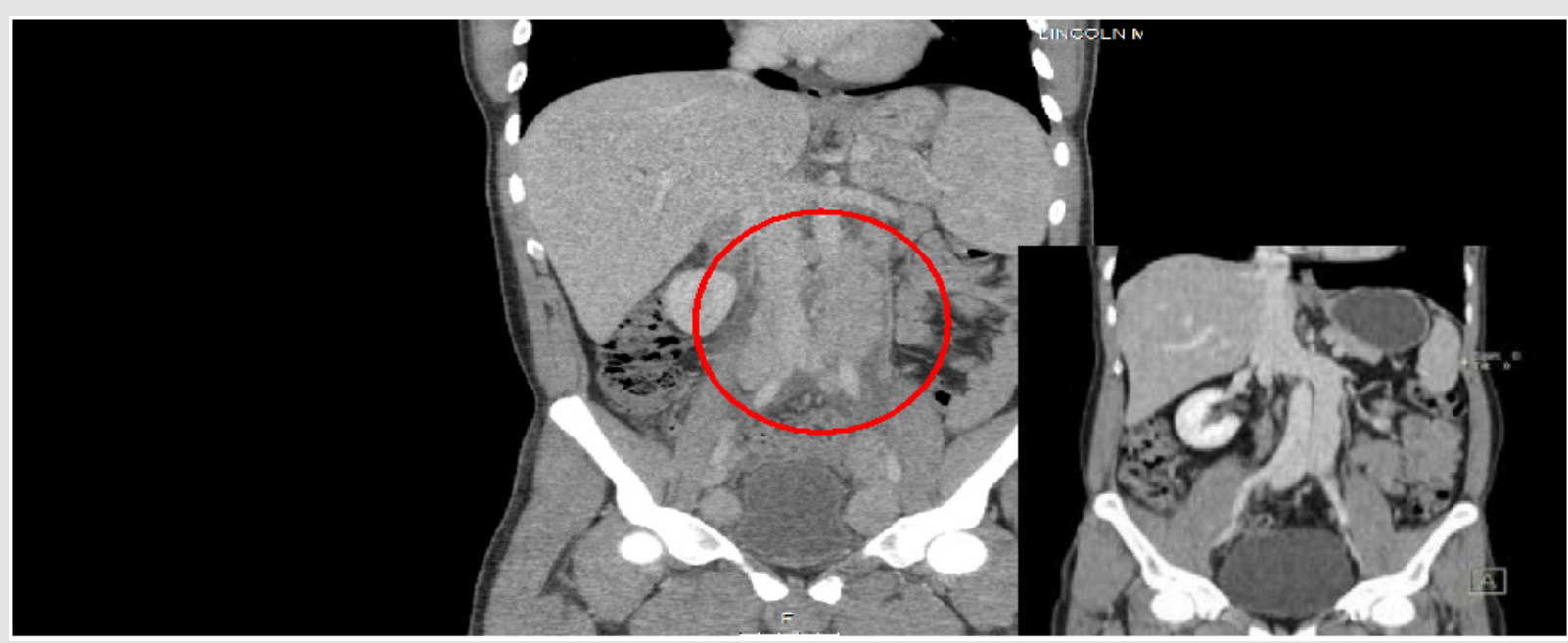

Figure 3.

Recent research has focused on FBXW7, a tumor suppressor gene that drives T-cell lymphoblastic neoplasia through its proteosomal degradation of proto-oncogenes. It is found on chromosome 4q32, a region often observed to be deleted in human tumors. Through alternative splicing, it expresses 3 isoforms which are responsible for the degradation of NOTCH1, c-myc, CCNE1, and SREBP1. Analysis of its role in T-LBL via DNA sequencing showed no functional mutational alterations, but frequent alterations observed in upstream factor-encoding genes that regulate the expression of FBXW7 (eg. CEP, TP53, REL, HESS, PSEN2). Analysis by RNA sequencing in the same study showed that FBXW7 was significantly downregulated in T-LBL samples, with additional deregulation of its regulator, TP53. Additionally, there was observed upregulation of specific microRNAs that silence FBXW7 in T-LBL samples, supporting the theory of the critical involvement of FBXW7 in T-LBL development [5]. Our patient clearly does not fit the typical picture of this disease. At 39 years old he is beyond the most common age of presentation. He tested negative for HTLV 1 and 2, HIV, and HCV, and his massive lymphadenopathy was atypical in location. He presented with B symptoms, elevated LDH, 
and bone marrow biopsy was negative. His age and the degree of spread at the time of presentation afford him a less than favorable prognosis, however with chemotherapeutic developments the cure rate is suggested to be $50 \%$ [6].

\section{Conclusion}

In view of the growing importance of molecular genetic analysis in the diagnosis and prognosis of T Cell Lymphoblastic Lymphoma/ Leukemia, it is important for hematologist and pathologist to use the routine histopathology, immunophenotyping, conventional cytogenetic analysis, fluorescence in situ hybridization and other tests, excellent resources to establish the diagnosis. However, a detailed clinical history and meticulous physical exam helps to identify the diagnosis.

\section{References}

1. Cortelazzo S, Ferreri A, Hoelzer D, Ponzoni M (2017) Lymphoblastic Lymphoma. Crti Rev Oncol Hematol 113: 304-317.

ISSN: 2574-1241

DOI: 10.26717/BJSTR.2019.21.003546

Cristabel Robles Hidalgo. Biomed J Sci \& Tech Res

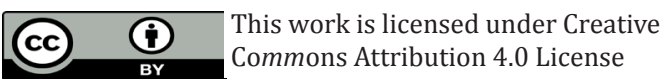

Submission Link: https://biomedres.us/submit-manuscript.php
2. You MJ, Medeiros LJ, Hsi ED (2015) T-lymphoblastic leukemia/ lymphoma. Am J Clin Pathol 144(3): 411-422.

3. Vazquez-Dominiguez I, Gonzalez-Sanchez L, Lopez-Nieva P, Pablo Fernández-Navarro, María Villa-Morales, María Á. Cobos-Fernández, et al. (2019) Downregulation of specific FBXW7 isoforms with differential effects in T-cell lymphoblastic lymphoma. Oncogene volume 38: 46204636.

4. Lepretre D, Graux C, Touzart A, Macintyre E, Boissei N, et al. (2017) Adult T-type lymphoblastic lymphoma: Treatment advances and prognostic indicators. Exp Hematol 51: 7-16.

5. Wang P, Peng X, Deng X, Gao L, Zhang X, et al. (2018) Diagnostic challenges in T-lymphoblastic lymphoma, early T-cell precursor acute lymphoblastic leukemia or mixed phenotype acute leukemia: A case report. 2018. Medicine (Baltimore) 97(41): e12743.

6. Wang XX, Wu D, Zhang L (2018) Clinical and molecular characterization of early T-cell precursor acute lymphoblastic leukemia: Two cases report and literature review. 2018. Medicine (Baltimore) 97(52): e13856.

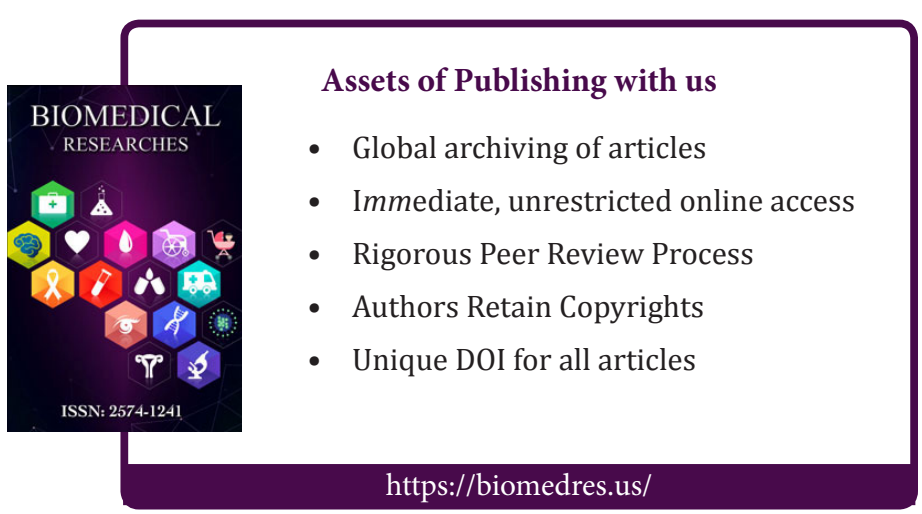

